\title{
Treatment of lower extremity fractures of the femur using a locked anatomical plate
}

Keywords: orthopedic treatment, screwing, femoral fractures

\section{Introduction}

Distal femoral fractures represent less than $1 \%$ of all fractures and $16.26 \%$ of all femoral fractures. As early as the 1970 s, surgical treatment took precedence over orthopedic treatment. ${ }^{1}$ A locked plate is defined as an osteosynthesis implant, the screws of which are fixed in the plate (regardless of mode of screwing: lock nut, ring, thread) and considered to be "internal fixators", 2,3

Their mechanical properties, ensure, among other things, better stability in fragile bones. ${ }^{4,5}$ In our case, the locked anatomical or condylar plate of the distal femur was largely indicated since it allows better stability of the fracture site, since most of the cases were closed fractures with comminution. The essential objectives of this surgery are, on the one hand the anatomical restitution of the knee joint and on the other hand early rehabilitation thanks to a stable fracture site. ${ }^{6}$ The aim of our work is to evaluate the results of using this locked plate in our department and compare with the data in the literature.

\section{Materials and method}

Our study was retrospective spread over a period of 4 years from January 2014 to January 2018, and which looks at 24 cases of fractures of the lower end of the femur (FLEF). All our cases were treated in the orthopedic and trauma surgery department A of HASSAN II teaching hospital in Fez surgically with a locked anatomical plate (Condylar plate LCP 4.5/5.0.). All patients over 16 years of age, admitted for fracture of the distal femur, who underwent osteosynthesis by locked anatomical plate during this period were included. The average age was 40 with extremes of 19 and 64; a male predominance at $67 \%$. The road traffic accidents on the public highway represented the most frequent etiology with 12 cases or $67 \%$. A high energy mechanism was found in the majority of our patients with $75 \%$ of cases. All of our patients were admitted initially in the emergency department. The clinical examination of our patients systematically included a vasculonervous examination in search of damage to the popliteal artery and the popliteal vein, to the sciatic nerve and its branches by observing the mobility of the foot and toes, and the peripheral pulses; posterior tibial, pedal and capillary. The radiological incidences done in our series for all our patients were AP and lateral views of the knee. A CT scan was requested for 2 of our patients. We used the AO classification for its simplicity.

All of our patients were operated on in strict lateral decubitus, using a lateral incision of the distal femur and under spinal anesthesia in most cases. The use of an image intensifier was necessary for 13 of our patients. All patients in our study benefited from osteosynthesis by a locked plate. No transplant was done in our series. A Redon drain was required in all cases for 48 hours. Immobilization for 5 to 8 weeks using a knee brace for all our patients. Low molecular weight heparin during the immobilization period and a 48-hour antibiotic prophylaxis were prescribed for all patients in our study.

We evaluated our results according to functional and anatomical criteria of VIVES: The anatomical criteria also made it possible to
Volume 12 Issue 3 - 2020

\author{
Lahrach K,' Soumare B,' Sidibe S,' Diarra S, ${ }^{2}$ \\ Saeed AR,' Boutayeb F' \\ 'Department of Traumatology and Orthopedics, Sidi \\ Mohammed Ben Abdellah University of Fez, Morocco \\ ${ }^{2}$ Laboratory of Epidemiology and Public Health, Sidi Mohammed \\ Ben Abdellah University of Fez, Morocco
}

Correspondence: Soumaré Boubacar, Resident Doctor in Traumatology and Orthopedics at the Hassan II University Hospital, Faculty of Medicine and Pharmacy, Sidi Mohammed Ben Abdellah University of Fez, Morocco, Tel 00212628815726/22376371922, Email docsoum19@gmail.com

Received: May 15, 2020 | Published: June 25, 2020

distinguish the results into 4 groups according to the radiological criteria of VIDAL AND MERCHAND (Normal axes, Defects of axes $<5^{\circ}$ frontal plane and $10^{\circ}$ sagittal plane, Deviation $10^{\circ}$ or $15^{\circ}$ sagittal plane or $10^{\circ}$ rotation, More than $15^{\circ}$ ). The functional rating is based on the SOFCOT score criteria (knee mobility, knee stability, pain, gait study).

\section{Results}

In our series, the fracture of the lower extremity of the femur involved both the right side with 13 cases and the left side with 10 cases with only one case of bilateral fracture. Radiological imaging remains an essential means of investigation carried out in all our patients. A CT scan was necessary in 2 of our patients due to the complexity of their fractures.

According to the SOFCOT classification, our study included 14 cases of extra articular fractures or 60\% (7 type I, 4 type II, 3 type III) while articular fractures were presented in 10 cases or $40 \%$ (3 type IV , 3 type V, 3 type VI, 1 type VII). According to that of AO, our study was dominated by the $\mathrm{C} 2$ type ( 8 cases) followed by the $\mathrm{A} 1$ type (6 cases). Skin lesions were classified according to Cauchoix and Duparc; type I in 4 cases and type II in 1 case.

We did not record any case of vascular or nerve lesions in our series. Eight patients in our series, who presented a lesional association of which 5 cases were poly fractures (A case of fracture of the upper extremity of the femur associated with an internal malleolus fracture, A case of associated fractures of the head of the 3rd 4th and 5 th metatarsal, A case of a floating knee with a fracture of the middle $1 \backslash 3$ and lower $1 \backslash 3$ junction of the homolateral tibial shaft, A fracture of the distal end of the radius) and 3 polytrauma patients: 2 with a head injury ( A case of head trauma without obvious bone or parenchymal lesions on the CT scan, and A second case presenting a left extradural hematoma operated immediately) and 1 case of abdominal trauma (a small hemoperitoneum and required strict monitoring). The vast majority of the patients in our series were operated on within a week, the average time before operating was 4 days, ranging from a minimum of 1 day to a maximum of 10 days. Depending on the 
lesions, 15 patients or $63 \%$ benefited from spinal anesthesia while 9 patients or $37 \%$ benefited from GA. In our series, all of our patients benefited from postoperative rehabilitation which was started after 6 weeks on average, as soon as the patient's condition permitted, but the times varied according to the type of fracture, and the degree of comminution.

The length of hospital stay was on average 6 days, ranging from a minimum of 2 days to a maximum of 18 days. The complications recorded were immediate, secondary and late: a single case of superficial infection in immediate postoperative, quickly suppressed by appropriate antibiotic therapy and daily dressing; a single case of secondary displacement in a 70-year-old patient after 5 months; 3 cases of delayed consolidation were reported, ie $12 \%$; One case of pseudarthrosis (35 years old with a stage V fracture of Sofcot and $\mathrm{C} 2$ of the $\mathrm{AO}$, associated with a stage I skin opening of cauchois and Duparc); 4 vicious calluses (2 cases of misalignment in varus, one greater than $5^{\circ}$ and a misalignment in Valgus and 1 case of shortening of $1 \mathrm{~cm}$ of the operated limb compared to the other member); 4 cases of knee stiffness were reported, representing $20 \%$ of cases including 2 cases of type IV of sofcot, 1 case of type $V$ of sofcot and 1 case of type VI of sofcot; and only 1 case of knee osteoarthritis. There were 3 cases of lateral instability of the knee during the 6th month of follow-up. No ligament or meniscal repair was made during the initial management.

Our functional results were: Very good: 9 cases, or $38 \%$, Good: 7 cases, or $29 \%$, Average: 6 cases, or $25 \%$, Poor: 2 cases, or $8 \%$, and $72 \%$ of the patients recovered a knee compatible with a normal working life with an average time for resumption of socio-professional activities of 6 months. Our anatomical results found were: Very good: 14 cases, or $58 \%$, Good: 4 cases, or $17 \%$, Average: 4 cases, or $17 \%$, Poor: 2 cases, or $8 \%$, and $89 \%$ of our patients recovered correct anatomical axis.

\section{Discussion}

The good anatomical and functional results linked to this type of assembly in our series have allowed and facilitated an evolution of operating techniques in our context with mechanical properties, ensuring, among other things, better resistance in fragile bone as approved by some authors. ${ }^{4,5}$

Age being an important prognostic factor, young people are the most affected in our series with an average age of 40 years unlike many western series where the average age is above 60 years this could be explained by the upsurge road accidents in our country and a shorter life expectancy.

Joint fractures were slightly predominant and accounted for 55\% of the cases in our series, with a dominance of type V fractures (20\%), types IV and VI presented $15 \%$ of cases, followed by type VII which presented $5 \%$ of cases; unlike many series which have reported a predominance of supra condylar fractures. ${ }^{7,8}$ Vascular-nerve lesions are rare, no cases listed in our series as corroborated by some authors. ${ }^{7,9}$ Menisco-ligaent lesions according to Ascencio ${ }^{9}$ were present in $20 \%$ of cases involving ACL and more rarely ELL. Although these lesions were not specifically looked for in our series, there is only 1 case of ACL lesion and 1 ELL case at 6 months follow-up. A skin opening in $21 \%$ of our patients correlates with certain series. ${ }^{10,11}$

In our series the mean intervention time, a prognostic factor was 4 days which lower than the results of many studies. ${ }^{11,12}$ The incision was lateral in our study with mechanical peculiarities evolving towards minimally invasive surgery ${ }^{2,3,13,14}$ and its many advantages.
A fairly low post-operative infection rate compared to certain series in the literature (POMBED LUC) indicating adequate and early management in our study.

Pseudarthrosis of the distal third of the femur should be relatively rare because it's an area rich in trabecular bone which has good osteogenic properties and whose vascular supply is excellent ${ }^{15}$ as corroborated by different studies., ${ }^{9,16,17}$ The vicious calluses with a relatively low rate according to the literature, ${ }^{16,17}$ were most due to surgery for failure fracture reduction or often of an early loading. Stiffness of the knee at a rate lower than $20 \%$ as in reported in literature. ${ }^{79}$ The insufficient number of follow-up in our series did not allow us to evaluate the results concerning osteoarthritis of the knee. For our functional results, $71 \%$ were good and very good results, which means our study is similar to that of other foreign series [SAW, WENG, RAHMI]. These results are comparable to those of other authors with reference to the ECMV [Iftikhar, Zaalane (ECM).

\section{Conclusion}

The locked anatomical plate thanks to its biomechanical peculiarities gives excellent results and offers perspectives towards minimally invasive surgery while retaining the biological advantages and stability.

\section{Acknowledgments}

None.

\section{Conflicts of interest}

The authors declare there are no conflicts of interest.

\section{Funding}

None.

\section{References}

1. Chiron P. Fractures of the lower end of the adult femur. EMC. 1995;52:147-166

2. Kregor PJ, Stannard JA, Zlowodzki M, et al. Treatment of distal femur fractures using the less invasive stabilization system: surgical experience and early clinical results in 103 fractures. J Orthop Trauma. 2004;18(8):509-520.

3. Ehlinger M, Adam P, Abane L, et al. Treatment of periprosthetic femoral fractures of the knee. Knee Surg Sport Trauma Arthros. 2011;19(9):1473-1478.

4. Perren SM. Evolution of the fixation of long bones fractures. The scientific basis of biological internal fixation: choosing a new balance between stability and biology. J Bone Joint Surg (B). 2002;84(8):10931110 .

5. Wagner M. General principles for the clinical use of the LCP. Injury. 2003;34(Suppl 2):31-42.

6. Müller ME, Allgöwer M, Schneider R, et al. AO Manual of Internal Fixation. 3rd Edition. Berlin: Springer; 1991:752.

7. Chun-Jui Weng, Chi-Chuan Wu, Kuo-Fun Feng, et al. Comparison of supraintercondylar and supracondylar femur fractures treated with condylar buttress plates. BMC Musculoskelet Disord. 2016;17(1):413.

8. Saw A, Lau CP. Supracondylar Nailing for Difficult Distal Femur Fractures. J Orthop Surg. 2003:11(2):141-147. 
9. Malawski S. Fractures of the lower end of the femur. Chir Narzadow Ruchu Ortop Pol. 1955;20(2):153-166.

10. Carson. European surgical orthopedics \& traumatology. 2014

11. The supra and inter condylar fractures of the femur in the Department of Orthopedic and Trauma Surgery of the HGT Faculty of Medicine, of pharmacy and odontostomatology. University of Bamako year academic: $2004-2005$.

12. Hammoumi Nasik. Anatomical plate versus centromedullary nailing of the femur. Trauma SCE and orthopedics B.

13. Helfet DL, Shonnard PY, Levine D, et al. Minimally invasive plate osteosynthesis of distal fractures of the tibia. Injury. 1997;28(suppl):42-47.
14. Ehlinger M, Brinkert D, Besse J, et al. Reversed anatomic distal femoral plate for periprosthetic hip fracture. Orthop Traumatol Surg Res. 2011;97(5):560-564.

15. Goldchild M, Vaz S, Nguyen T, et al. Fractures of the lower extremity of the femur in adults. Ann Orthop West. 1999;31:219-246.

16. Ely L Steinberg, Jacov Elis, Yohai Steinberg, et al. A doubleplating approach to distal femur fracture: A clinical study. Injury. 2017;48(10):2260-2265.

17. Sherif G. Dynamic locked plating for fixation of distal femur fractures using near- cortical over-drilling: Preliminary results of a prospective observational study. J Clin Orthop Trauma. 2017;8(3):215-219. 